Accepted version of the article published in IEEE International Conference on Acoustics, Speech and Signal Processing (ICASSP), 2021. DOI: 10.1109/ICASSP39728.2021.9415032. Available at: http://ieeexplore.iee.org

\title{
AFFINE PROJECTION SUBSPACE TRACKING
}

\author{
Marc Vilà, Carlos Alejandro López, Student Members, IEEE, and Jaume Riba, Senior Member, IEEE
Signal Theory and Communications Department, Technical University of Catalonia (SPCOM/UPC) \{marc.vila.insa, carlos.alejandro.lopez, jaume.riba\}@upc.edu

\begin{abstract}
In this paper, we consider the problem of estimating and tracking an R-dimensional subspace with relevant information embedded in an $\mathbf{N}$-dimensional ambient space, given that $\mathbf{N}>>\mathbf{R}$. We focus on a formulation of the signal subspace that interprets the problem as a least squares optimization. The approach we present relies on the geometrical concepts behind the Affine Projection Algorithms (APA) family to obtain the Affine Projection Subspace Tracking (APST) algorithm. This on-line solution possesses various desirable tracking capabilities, in addition to a high degree of configurability, making it suitable for a large range of applications with different convergence speed and computational complexity requirements. The APST provides a unified framework that generalises other well-known techniques, such as Oja's rule and stochastic gradient based methods for subspace tracking. This algorithm is finally tested in a few synthetic scenarios against other classical adaptive methods.
\end{abstract}

Index Terms - On-line Subspace Learning (OSL), Affine Projection Subspace Tracking (APST), Affine Projection Algorithms (APA), Projection Approximation Subspace Tracking (PAST)

\section{INTRODUCTION}

A common issue present in many applications of signal processing and machine learning is dealing with high-dimensional data: whether it be internet traffic or sensor networks, the current trend is having to face progressively larger data sets, translating into higher memory storage and computational power requirements. It is then a question of capital importance to process such data groups to make them easier to handle.

A fundamental breakthrough in solving this limitation was the idea that relevant information in a data set usually manifests itself in an underlying structure of much lower dimensionality than the ambient space. Since such mathematical entity is often a subspace, the field of signal processing that exploits this concept is known as subspace learning, initiated with the introduction of Principal Component Analysis (PCA) [1]. From that point onwards, there has appeared a plethora of methods to efficiently estimate and track signal subspaces in ambient spaces. Many of them operate on batch mode, i.e., they have to store all the available data to process them at once, which does not solve the problem of limited memory and computational power. On the contrary, On-line Subspace Learning (OSL) reduces the use of resources and enables to continuously track changes onto the subspace at the expense of estimation precision.

This work has been supported by the Spanish Ministry of Science and Innovation through project RODIN (PID2019-105717RB-C22 / AEI / $10.13039 / 501100011033$ ) and by the Catalan Government (AGAUR) under grant 2017 SGR 578.
Low-cost adaptive solutions have been typically used in direction of arrival (DOA) estimation [2-5], although there has recently been an effort to import these ideas into pure communications fields (CDMA, MIMO, etc.) [6-8]. To concretely motivate our research, we give an example of a novel application of OSL in noncoherent communications over a block-fading channel [9]. In such scenario at high SNR, it is shown in [10] that the orientation of a received signal vector within a subspace is irrelevant, since the object that transmits information is the subspace itself. These insights open the door to the design of signal subspaces and a way to estimate them on-line using the resources available by not having channel state information (CSI), the main characteristic of noncoherent communications.

The work presented here has focused on the formulation of the Affine Projection Subspace Tracking (APST) algorithm, a novel method for OSL. It is based on some key concepts taken from the work by B. Yang [11] regarding the problem of tracking a subspace adaptively. While the author considers a recursive least squares $(R L S)$ solution, we depart from such approach by applying the ideas and geometrical foundations behind the Affine Projection Algorithms (APA) family. This is a notable group of adaptive algorithms to solve least squares problems that provides an adjustable compromise between the simplicity of least mean squares (LMS) solutions and the convergence speed of RLS-based ones. By reusing past data samples, it offers some desirable tracking properties such as robustness against very autocorrelated signals. Although the APA family is still underexploited in many subfields of signal processing, its applications are currently flourishing thanks to major works like [12], by K. Ozeki. With this paper we aim at contributing to that proliferation.

\section{PROBLEM STATEMENT}

Let $\mathbf{x}[k] \in \mathbb{C}^{N}$ be a data vector observed at the $k$ th snapshot, obtained from the following model:

$$
\mathbf{x}[k] \triangleq \sum_{r=1}^{R} s_{r}[k] \mathbf{h}_{r}[k]+\mathbf{n}[k]=\mathbf{H}[k] \mathbf{s}[k]+\mathbf{n}[k],
$$

where $\mathbf{H}[k] \triangleq\left[\mathbf{h}_{1}[k] \ldots \mathbf{h}_{R}[k]\right] \in \mathbb{C}^{N \times R}$ is a deterministic matrix sequence and $\mathbf{s}[k] \triangleq\left[s_{1}[k] \ldots s_{R}[k]\right]^{T} \in \mathbb{C}^{R}$ is a random source vector with correlation matrix $\mathbf{C}_{\mathbf{s}} \triangleq \mathrm{E}\left[\mathbf{s}[k] \mathbf{s}^{T}[k]\right]$ (E[.] denotes the expectation operator). $R$ is assumed to be known. $\mathbf{n}[k]$ is a noise component added to the observation vector and uncorrelated with $\mathbf{s}[k]$, which will be considered AWGN, $\mathbf{n}[k] \sim \mathcal{C N}\left(\mathbf{0}, \sigma_{n}^{2} \mathbf{I}_{N}\right)$.

For ease of notation, in this section we will set $\mathbf{H}[k]$ to be constant $(\mathbf{H}[k] \triangleq \mathbf{H})$. Let $\mathbf{C}_{\mathbf{x}}$ be the observation correlation matrix, defined and eigendecomposed as:

$$
\mathbf{C}_{\mathbf{x}} \triangleq \mathrm{E}\left[\mathbf{x}[k] \mathbf{x}^{H}[k]\right]=\mathbf{H} \mathbf{C}_{\mathbf{s}} \mathbf{H}^{H}+\sigma_{n}^{2} \mathbf{I}_{N} \triangleq \mathbf{U} \boldsymbol{\Sigma} \mathbf{U}^{H},
$$

where $\boldsymbol{\Sigma} \triangleq \operatorname{diag}\left(\lambda_{1}, \ldots, \lambda_{N}\right)$ are the eigenvalues and $\mathbf{U} \triangleq$ $\left[\mathbf{u}_{1}, \ldots, \mathbf{u}_{N}\right]$ are their corresponding orthonormal eigenvectors.

(C2021 IEEE. Personal use of this material is permitted. Permission from IEEE must be obtained for all other uses, in any current or future media, including reprinting/republishing this material for advertising or promotional purposes, creating new collective works, for resale or redistribution to servers or lists, or reuse of any copyrighted component of this work in other works.

DOI: 10.1109/ICASSP39728.2021.9415032 
Given that $R<N$, the eigenvalues can be ordered as follows:

$$
\underbrace{\lambda_{1} \geq \cdots \geq \lambda_{R}}_{\text {Signal eigenvalues }}>\underbrace{\lambda_{R+1}=\cdots=\lambda_{N}}_{\text {Noise eigenvalues }}=\sigma_{n}^{2} .
$$

The first $R$ dominant eigenvalues and their associated eigenvectors correspond to the signal subspace, while the $N-R$ last eigenpairs are related to the noise one. $\mathbf{U}$ can then be split into two column spans: $\mathbf{U} \triangleq\left[\mathbf{U}_{\mathbf{s}} \mid \mathbf{U}_{\mathbf{n}}\right]=\left[\mathbf{u}_{1} \ldots \mathbf{u}_{R} \mid \mathbf{u}_{R+1} \ldots \mathbf{u}_{N}\right]$, which define the signal and noise subspaces, orthogonal with each other. $\mathbf{U}_{\mathbf{s}}$ spans the same subspace as $\mathbf{H}$.

In many applications, the eigenvectors are not required; just finding an equivalent basis $\mathbf{W}$ suffices to define the signal subspace. This is the approach we will take in following sections.

\subsection{Alternative signal subspace interpretation}

The previous scenario can be tackled from various points of view. In this paper we will take advantage of understanding it as an optimization problem. Let us define the following scalar cost function:

$$
J(\mathbf{W}[k]) \triangleq \mathrm{E}\left[\left\|\mathbf{x}[k]-\mathbf{W}[k] \mathbf{W}^{H}[k] \mathbf{x}[k]\right\|^{2}\right],
$$

which takes the argument $\mathbf{W}[k] \in \mathbb{C}^{N \times R}$. In [11], the author proves that $J$ has a global minimum which is reached if and only if $\mathbf{W}[k]=\mathbf{U}_{\mathbf{s}} \mathbf{Q}$, where $\mathbf{Q} \in \mathbb{C}^{R \times R}$ is unitary. In other words, by minimizing $J(\mathbf{W}[k])$ we can converge to an orthonormal basis of the signal subspace of $\mathbf{x}[k]$. This alternative interpretation of the subspace tracking problem will allow us to apply gradient based and adaptive estimation techniques.

Since the statistics of the observed model are unknown, we will replace the expectation from $J(\mathbf{W}[k])$ with an estimation based on a sliding window, defined as:

$$
\begin{aligned}
\hat{J}(\mathbf{W}[k]) & \triangleq \frac{1}{P} \sum_{p=0}^{P-1}\left\|\mathbf{x}[k-p]-\mathbf{W}[k] \mathbf{W}^{H}[k] \mathbf{x}[k-p]\right\|^{2} \\
& =\frac{1}{P}\left\|\mathbf{X}[k]-\mathbf{W}[k] \mathbf{W}^{H}[k] \mathbf{X}[k]\right\|_{F}^{2},
\end{aligned}
$$

where $\mathbf{X}[k] \triangleq[\mathbf{x}[k] \ldots \mathbf{x}[k-P+1]]$ is called regressor block of size $P$.

\subsection{Projection approximation}

Equation (5) is a fourth-order function of $\mathbf{W}[k]$, which makes it remarkably complex to optimize adaptively, since deciding its convexity is an NP-hard problem [13]. Referring once again to [11], the author proposes a simple modification of $\hat{J}(\mathbf{W}[k])$ that can reduce noticeably the task of minimizing it. Instead of using $\mathbf{W}^{H}[k] \mathbf{x}[k-p]$, which corresponds to the projections of $\mathbf{x}[k-p]$ onto the columns of $\mathbf{W}[k]$, Yang defines the usage of the vectors

$$
\mathbf{y}[k-p] \triangleq \mathbf{W}^{H}[k-1] \mathbf{x}[k-p], p=0, \ldots, P-1 .
$$

Substituting $\mathbf{W}^{H}[k] \mathbf{x}[k-p]$ in (5) produces

$$
\begin{aligned}
\hat{J}^{\prime}(\mathbf{W}[k]) & \triangleq \frac{1}{P} \sum_{p=0}^{P-1}\|\mathbf{x}[k-p]-\mathbf{W}[k] \mathbf{y}[k-p]\|^{2} \\
& =\frac{1}{P}\|\mathbf{X}[k]-\mathbf{W}[k] \mathbf{Y}[k]\|_{F}^{2},
\end{aligned}
$$

where $\mathbf{Y}[k] \triangleq \mathbf{W}^{H}[k-1] \mathbf{X}[k]=[\mathbf{y}[k] \ldots \mathbf{y}[k-P+1]] \in$ $\mathbb{C}^{R \times P}$. This new function $\hat{J}^{\prime}$ is quadratic with respect to $\mathbf{W}[k]$. Additionally, when dealing with stationary or slowly varying signals, the error of approximating $\mathbf{W}^{H}[k] \mathbf{X}[k]$ with $\mathbf{W}^{H}[k-1] \mathbf{X}[k]$ becomes minimal. Therefore, we can expect the optimization of $\hat{J}^{\prime}$ to be a good estimate of the signal subspace.

\section{METHODOLOGY}

The key idea to solve the subspace learning problem from Section 2 with an Affine Projection Algorithm is the following. Notice that (7) is a residual sum of squares which, once minimized, provides an approximate solution to the system of linear equations:

$$
\mathbf{W}[k] \mathbf{Y}[k]=\mathbf{X}[k] \stackrel{\text { dual }}{\Longleftrightarrow} \mathbf{Y}^{H}[k] \mathbf{W}^{H}[k]=\mathbf{X}^{H}[k] .
$$

In this context, matrix $\mathbf{Y}[k]$ defines the linear map $\mathbf{Y}[k]: \mathbb{C}^{P} \rightarrow$ $\mathbb{C}^{R}$ and $\mathbf{Y}^{H}[k]: \mathbb{C}^{R} \rightarrow \mathbb{C}^{P}$ is its dual. We will refer to either side of (8) correspondingly for a clearer notation and geometric meaning.

If the optimal solution of (8), $\mathbf{W}_{o p t}^{H}$, exists, it has to fulfill the relations $\mathbf{y}^{H}[k-p] \mathbf{W}_{o p t}^{H}=\mathbf{x}^{H}[k-p]$ for $p=0, \ldots, P-1$. Each one of these restrictions determines a geometric structure called affine subspace [12], expressed as:

$$
\Pi_{k-p} \triangleq\left\{\mathbf{W}^{H}[k] \in \mathbb{C}^{R \times N} ; \mathbf{y}^{H}[k-p] \mathbf{W}^{H}[k]=\mathbf{x}^{H}[k-p]\right\},
$$

where $\{\cdot\}$ denotes the set of all $\mathbf{W}^{H}[k]$ that fulfill that condition. Since there are $P$ constraints, $\mathbf{W}_{o p t}^{H}$ has to belong to the $P$ affine subspaces at once. This means it must be found in their intersection $\Pi^{(k)} \triangleq \bigcap_{p=0}^{P-1} \Pi_{k-p}$, which is also an affine subspace and remains unaffected by their relative orientation. Exploiting this property results in desirable convergence features when tracking on very autocorrelated signals [12].

In an adaptive filtering scheme, we want to approach $\mathbf{W}_{\text {opt }}$ progressively. At every iteration $k$, the shortest path to approach the optimal solution follows the projection of the current estimate onto the intersection of subspaces, where $\mathbf{W}_{o p t}^{H}$ is found. This projection is expressed as $\mathcal{P}_{\Pi^{(k)}}\left(\mathbf{W}^{H}[k]\right)$ or as $\mathcal{P}_{\Pi^{(k)}}(\mathbf{W}[k])$ in its dual form. To do so, we define the next update equation:

$$
\mathbf{W}[k+1]=\mathbf{W}[k]+\mu \overbrace{\left(\mathcal{P}_{\Pi^{(k)}}(\mathbf{W}[k])-\mathbf{W}[k]\right)}^{\Delta \mathbf{W}[k]} .
$$

where $\mu>0$ is called step-size and $\Delta \mathbf{W}[k]$ is the direction of change.

It is known [12] that, when a solution for (8) exists, $\Pi^{(k)}$ contains it. Even when there is no solution, such affine subspace contains the set of least squares approximations, i.e., the set of $\mathbf{W}[k]$ that minimize (7). Thus, this set can alternatively be defined as:

$$
\Pi^{(k)} \triangleq\left(\mathbf{X}[k] \mathbf{Y}^{+}[k]\right)^{H}+\operatorname{ker}\left(\mathbf{Y}^{H}[k]\right),
$$

where $\left(\mathbf{X}[k] \mathbf{Y}^{+}[k]\right)^{H}$ is the minimum norm solution of the system. $\operatorname{ker}(\cdot)$ denotes the null space and $\mathbf{Y}^{+}[k]$ is the Moore-Penrose pseudoinverse of $\mathbf{Y}[k]$, which fulfills $\mathbf{Y}^{+}[k] \mathbf{Y}[k]=\mathbf{I}_{P}$. The geometrical interpretation of (11) is the following: $\Pi^{(k)}$ is an affine subspace parallel to $\operatorname{ker}\left(\mathbf{Y}^{H}[k]\right)$ and displaced by $\left(\mathbf{X}[k] \mathbf{Y}^{+}[k]\right)^{H}$. This displacement vector is an element of the orthogonal complement of the null space of $\mathbf{Y}^{H}[k], \operatorname{ker}\left(\mathbf{Y}^{H}[k]\right)^{\perp}$, or, equivalently, it belongs to the range space of $\mathbf{Y}[k], \operatorname{ran}(\mathbf{Y}[k])$. With the well known decomposition $\mathbb{C}^{R}=\operatorname{ker}\left(\mathbf{Y}^{H}[k]\right) \oplus \operatorname{ran}(\mathbf{Y}[k])$, we can express $\mathbf{W}[k]$ as a function of the projections onto $\Pi^{(k)}$ and onto $\operatorname{ran}(\mathbf{Y}[k])$ :

$$
\begin{aligned}
\mathbf{W}[k] & =\mathcal{P}_{\Pi^{(k)}}(\mathbf{W}[k])-\left(\mathbf{X}[k] \mathbf{Y}^{+}[k]-\mathbf{W}[k] \mathbf{P}_{\operatorname{ran}(\mathbf{Y}[k])}\right) \\
& =\mathcal{P}_{\Pi^{(k)}}(\mathbf{W}[k])-\left(\mathbf{X}[k] \mathbf{Y}^{+}[k]-\mathbf{W}[k] \mathbf{Y}[k] \mathbf{Y}^{+}[k]\right) .
\end{aligned}
$$

Notice the term $\mathbf{Y}[k] \mathbf{Y}^{+}[k]$ is the projection matrix onto the subspace spanned by the columns of $\mathbf{Y}[k]$. By rearranging (12), we can get an expression for the projection of $\mathbf{W}[k]$ onto $\Pi^{(k)}$.

$$
\mathcal{P}_{\Pi^{(}(k)}(\mathbf{W}[k])=\mathbf{W}[k]+(\mathbf{X}[k]-\mathbf{W}[k] \mathbf{Y}[k]) \mathbf{Y}^{+}[k] .
$$


If we combine this result into (10), we obtain

$$
\mathbf{W}[k+1]=\mathbf{W}[k]+\mu(\mathbf{X}[k]-\mathbf{W}[k] \mathbf{Y}[k]) \mathbf{Y}^{+}[k] .
$$

We can further simplify this expression by defining the error matrix $\mathbf{E}[k] \triangleq \mathbf{X}[k]-\mathbf{W}[k] \mathbf{Y}[k]$, which leaves us with the basic update equation of the APST:

$$
\mathbf{W}[k+1]=\mathbf{W}[k]+\mu \mathbf{E}[k] \mathbf{Y}^{+}[k]
$$

We may relate this expression to various other adaptive algorithms. On the one hand, we can view the APST as a generalization of Oja's learning rule [11], a classical technique for PCA. Studying the simplest case possible $(R=1$ and $P=1)$, (15) becomes almost identical to Oja's rule:

$$
\mathbf{w}[k+1]=\mathbf{w}[k]+\mu \frac{y^{*}[k]}{\|y[k]\|^{2}}(\mathbf{x}[k]-y[k] \mathbf{w}[k]) .
$$

The only difference is the step size: while Oja's rule uses a fixed finely tuned $\mu$ [11], (16) provides a power normalized step-size that achieves better convergence [11]. On the other hand, the $P=1$ case transforms into a stochastic gradient descent method to minimize (5), akin to the normalized LMS (NLMS):

$$
\mathbf{W}[k+1]=\mathbf{W}[k]+\frac{\mu}{\|\mathbf{y}[k]\|^{2}}(\mathbf{x}[k]-\mathbf{W}[k] \mathbf{y}[k]) \mathbf{y}^{H}[k] .
$$

The most relevant conclusion about these results is the following. In classical estimation, LMS and RLS represent the two ends of a complexity/speed spectrum, while APA lays in between. Concurrently, in OSL, stochastic gradient descent methods and PAST [11] hold those positions, but no algorithm was analogous to APA until now. From the previous relations we see that the APST fills this gap and binds the two estimation fields into one interconnected landscape, while opening the door to a new subgroup of adaptive solutions.

\subsection{Regularization}

In (15), $\mathbf{Y}^{+}[k]$ is computed as $\mathbf{Y}^{+}[k] \triangleq\left(\mathbf{Y}^{H}[k] \mathbf{Y}[k]\right)^{-1} \mathbf{Y}^{H}[k]$, assuming $P<R$ and $\mathbf{Y}[k]$ has full column rank. Inverting the Gramian matrix $\mathbf{Y}^{H}[k] \mathbf{Y}[k]$ entails computational issues if it is illconditioned. To overcome this limitation, we apply a technique known as Tikhonov regularization [12]. The previous Gramian matrix becomes $\left(\mathbf{Y}^{H}[k] \mathbf{Y}[k]+\delta \mathbf{I}_{P}\right)$, which is always invertible, even when $\mathbf{Y}^{H}[k] \mathbf{Y}[k]$ is not. $\delta$ is a small positive number called regularization factor. After regularization, (15) becomes

$$
\mathbf{W}[k+1]=\mathbf{W}[k]+\mu \mathbf{E}[k]\left(\mathbf{Y}^{H}[k] \mathbf{Y}[k]+\delta \mathbf{I}_{P}\right)^{-1} \mathbf{Y}^{H}[k] .
$$

\subsection{Computational complexity}

Computing (18) entails a complexity of $O(R N P)+O\left(N P^{2}\right)+$ $O\left(P^{3}\right)$, which is beneficial for applications where the signal dimension is large $(R>>P)$. Terms $O\left(N P^{2}\right)$ and $O\left(P^{3}\right)$ are mostly affected by the projection order, which is a configurable parameter and not imposed by the studied problem. In some cases, however, we may want to increase the projection order $(P>>R)$ to obtain higher convergence speed. We can reduce the complexity of the algorithm by applying the Woodbury inversion lemma to (18), which leaves us with the equivalent

$$
\mathbf{W}[k+1]=\mathbf{W}[k]+\mu \mathbf{E}[k] \mathbf{Y}^{H}[k]\left(\mathbf{Y}[k] \mathbf{Y}^{H}[k]+\delta \mathbf{I}_{R}\right)^{-1}
$$

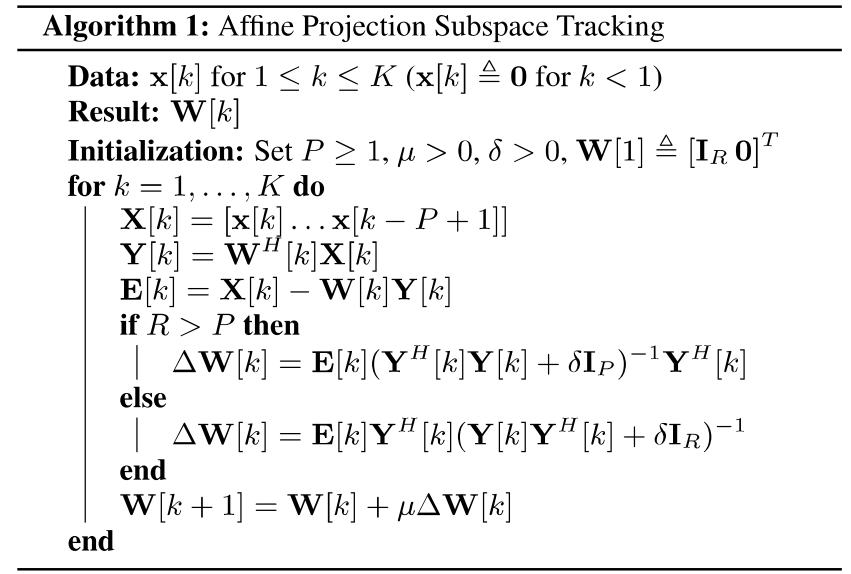

This new expression can replace (18) and has a complexity of $O(R N P)+O\left(N R^{2}\right)+O\left(R^{3}\right)$. It now depends heavier on the signal dimension, letting us increase $P$ without significant computational cost. Algorithm 1 implements the APST update equation considering both regularization and reduction of complexity.

\section{NUMERICAL RESULTS}

\subsection{Performance metrics}

Before displaying some numerical simulations to highlight the applicability of the APST, we will introduce the figure with which its performance will be tested.

The goal of the algorithm is to estimate a basis for the signal subspace of $\mathbf{x}[k]$, without any restriction like orthonormality. It is then reasonable to consider a metric that takes the same value for equivalent subspace bases, i.e., it is invariant to scaling and rotation. Our choice is the Projection Frobenius Distance, which is already widely used in the literature $[9,14,15]$. Given the true basis of the signal subspace, $\mathbf{H}[k]$, and an estimated one, $\mathbf{W}[k]$, their orthogonal projectors are defined as $\mathbf{P}_{\mathbf{H}[k]} \triangleq \mathbf{H}[k] \mathbf{H}^{+}[k]$ and $\mathbf{P}_{\mathbf{W}[k]} \triangleq$ $\mathbf{W}[k] \mathbf{W}^{+}[k]$, respectively. The Projection F-Distance between the subspaces they span is then defined as:

$$
d_{P F}(\mathbf{H}[k], \mathbf{W}[k]) \triangleq \frac{1}{\sqrt{2}}\left\|\mathbf{P}_{\mathbf{H}[k]}-\mathbf{P}_{\mathbf{W}[k]}\right\|_{F}=\|\sin (\boldsymbol{\theta})\|_{2},
$$

where $\boldsymbol{\theta} \triangleq\left[\theta_{1} \ldots \theta_{R}\right]^{T}$ are the principal angles between the two subspaces [15]. Notice that $0 \leq d_{P F} \leq \sqrt{R}$.

To have some performance references, the APST will be tested against three well-known algorithms: the stochastic gradient descent (GRAD) algorithm described in [11], the Exponentially Weighted PAST (PAST) [11] and the Sliding Window PAST (SW-PAST) [16]. They have been chosen to have an analogy of how the APA is usually tested against the LMS and the RLS. We use two versions of PAST to have fairer comparisons in non-stationary scenarios.

\subsection{Global configuration}

We use the same model as in (1), with $N \triangleq 10$ and $R \triangleq 3$. The signal is $\mathbf{s}[k] \sim \mathcal{C N}\left(\mathbf{0}, \mathbf{I}_{R}\right)$ and the noise $\mathbf{n}[k] \sim \mathcal{C N}\left(\mathbf{0}, 10^{-4} \times \mathbf{I}_{N}\right)$. $K \triangleq 3 \times 10^{4}$ time samples will be taken (using more samples results in numerical divergences in PAST caused by rounding errors [11]). The algorithms will attempt to estimate a matrix $\mathbf{W}[k]$ which spans the same subspace as $\mathbf{H}[k]$. The results displayed will be averaged over 5 simulations. 
The configuration of the four algorithms is the following. The forgetting factor of the PAST is $\beta \triangleq 0.999$, while the step-size of the GRAD is $\mu_{G R A D} \triangleq 1-\beta=10^{-3}$. The sliding window of the SWPAST has a length of $L_{W} \triangleq \frac{2}{1-\beta}=2000$ to provide comparable error floor levels. The step size and projection order of the APST is $\mu_{A P S T} \triangleq 10^{-3}$ and $P \triangleq 30$ unless stated otherwise.

\subsection{Stationary subspace}

In the first scenario, a stationary setting is considered $(\mathbf{H}[k] \triangleq \mathbf{H})$ to test the APST with different $P$ values (Fig. 1). The step-size of the APST has been tuned for each $P$ to obtain the same error floor, so their convergence speeds are comparable. The condition number of the autocorrelation of $\mathbf{x}[k]$ is of the order of 175 .

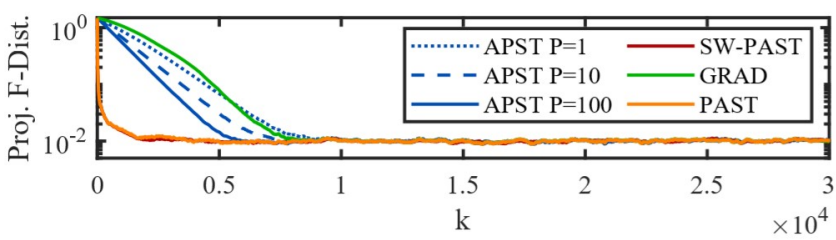

Fig. 1: Comparison between PAST, SW-PAST, GRAD and APST with various projection order values.

For $P \triangleq 1\left(\mu_{A P S T} \triangleq 2.05 \times 10^{-3}\right)$, the APST approaches the real subspace the slowest. Going from no intersection, i.e., $P \triangleq 1$, to a projection order of $10\left(\mu_{A P S T} \triangleq 8 \times 10^{-4}\right)$ produces a significant improvement in speed. Further augmenting $P$ to $100\left(\mu_{A P S T} \triangleq\right.$ $10^{-3}$ ) results in a faster convergence, although the improvement from 10 to 100 is similar to the one from 1 to 10 .

\subsection{Smooth change}

This scenario tests the tracking capabilities of the APST. During the first 10000 samples, the setting will remain stationary $(\mathbf{H}[k] \triangleq \mathbf{H})$. Once that instant is reached, $\mathbf{H}[k]$ changes progressively so that the subspace it spans varies gradually by following a geodesic with a random direction onto the Grassmannian $\mathbf{G r}(R, N)$ (see [15] for a complete explanation of this concept). The amount the subspace moves along the geodesic at each time instant is controlled by the step length $\Delta_{m o v}$. Two values of $\Delta_{m o v}$ will be used to observe how the algorithm reacts to slow and fast tracking requirements. Fig. 2 is a plot of the amount the subspace has moved at each time instant in the two tests, while Fig. 3 contains the corresponding estimation error of every algorithm in each case.

In the slow case, the four algorithms present similar tracking capabilities. The differences, however, appear in the fast one. GRAD has the highest tracking error, while SW-PAST has the lowest, which is expected since it uses a rectangular window instead of an exponential forgetting factor. PAST and APST present almost the same tracking error level, slightly above the one from SW-PAST.

\subsection{Sudden change}

The final test consists in a stationary matrix that, at a certain time instant, suffers a sudden change in all its values: $\mathbf{H}[k] \triangleq \mathbf{H}_{1}$ for $0<k \leq 15000$ and $\mathbf{H}[k] \triangleq \mathbf{H}_{2}$ for $15000<k \leq 30000$, such that $d_{P F}\left(\mathbf{H}_{1}, \mathbf{H}_{2}\right) \approx 1.2548$, a value close to the maximum distance for $R=3$.

This scenario emphasizes the strengths of the APST. Looking at Fig. 4, when the subspace change occurs, SW-PAST starts tracking it the fastest, but it then settles at a higher error level than the rest. PAST estimates the new subspace much slower than it does the first:
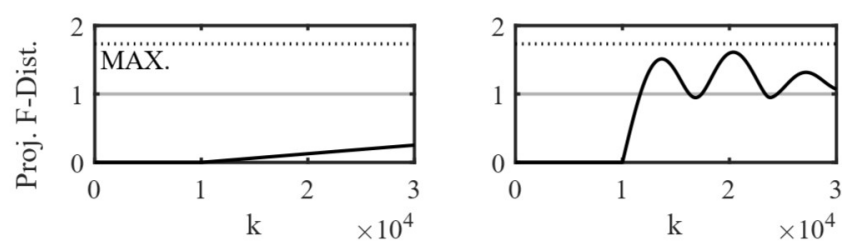

Fig. 2: Projection F-Distance between the original subspace and the displaced one at each time instant. $\Delta_{m o v}$ is $1 \times 10^{-5}$ in the left plot and $5 \times 10^{-4}$ in the right one.
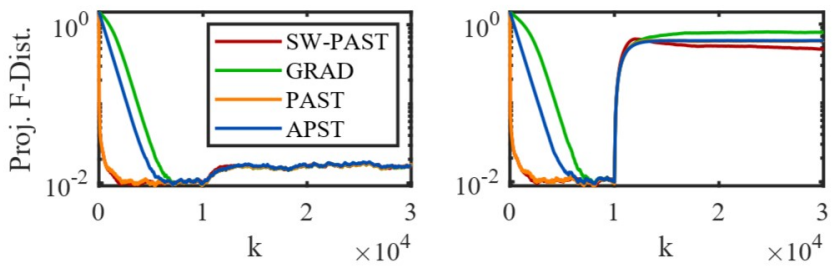

Fig. 3: Comparison between SW-PAST, PAST, GRAD and APST in the subspace tracking environments of Fig. 2.

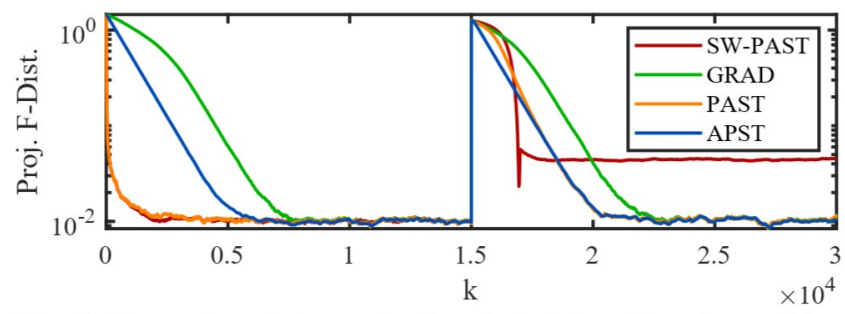

Fig. 4: Comparison between the four tested algorithms in a sudden change scenario.

its rate decreases to the speed of the APST. GRAD is the slowest, but its rate is consistent with the first subspace estimation. The same occurs with APST, which is the best of the four in terms of robustness and convergence speed, since its performance and numerical stability are not affected by a sudden change on the observations.

\section{CONCLUSIONS}

In this paper we proposed a novel on-line algorithm for subspace learning: the APST. We took the subspace problem interpretation presented in [11] and showed it can be solved by using the ideas behind the design of the Affine Projection Algorithms [12]. With this methodology, we obtained an adaptive and highly configurable solution, that provides reasonable tracking capabilities and robustness against sudden subspace changes and numerical precision limitations. In addition, it constitutes a framework that encompasses other well-known OSL methods.

The APST is based on a straightforward form of APA and leaves room for further improvements. From the subspace estimation point of view, it can incorporate orthonormalization mechanisms already used in the field $[16,17]$. In order to reduce the computational complexity requirements, the structure of the APST update equation can be exploited in several ways, similarly to how it is done in many variations of the APA, compiled in [12]. Regarding convergence speed, there exist variations of the APA that use variable step-sizes and projection orders [18], ideas that can be borrowed in subspace tracking as well. The insights presented in this work contribute to the landscape of OSL and may open the door to new lines of research. 


\section{REFERENCES}

[1] T. Bouwmans, N. Vaswani, P. Rodriguez, R. Vidal, and Z. Lin, "Introduction to the Issue on Robust Subspace Learning and Tracking: Theory, Algorithms, and Applications," IEEE Journal of Selected Topics in Signal Processing, vol. 12, no. 6, pp. 1127-1130, December 2018

[2] J. Xin and A. Sano, "Efficient Subspace-Based Algorithm for Adaptive Bearing Estimation and Tracking," IEEE Transactions on Signal Processing, vol. 53, no. 12, pp. 4485-4505, December 2005.

[3] B. Liao, Z. G. Zhang, and S. C. Chan, "A New Robust Kalman Filter-Based Subspace Tracking Algorithm in an Impulsive Noise Environment," IEEE Transactions on Circuits and Systems II: Express Briefs, vol. 57, no. 9, pp. 740-744, September 2010.

[4] B. Jalal, X. Yang, X. Wu, T. Long, and T. K. Sarkar, "Efficient Direction-of-Arrival Estimation Method Based on VariableStep-Size LMS Algorithm," IEEE Antennas and Wireless Propagation Letters, vol. 18, no. 8, pp. 1576-1580, August 2019.

[5] V. Nguyen, K. Abed-Meraim, N. Linh-Trung, and R. Weber, "Generalized Minimum Noise Subspace for Array Processing," IEEE Transactions on Signal Processing, vol. 65, no. 14, pp. 3789-3802, July 2017.

[6] I. Ali, D. N. Kim, and T. T. Jeong, "A New Subspace Tracking Algorithm Using Approximation of Gram-Schmidt Procedure," 2009 International Conference on Information and Multimedia Technology, pp. 244-248, 2009.

[7] M. Huang, X. Chen, S. Zhou, and J. Wang, "Low-complexity Subspace Tracking Based Channel Estimation Method for OFDM Systems in Time-Varying Channels," 2006 IEEE International Conference on Communications, vol. 10, pp. 46184623, 2006

[8] S. Buzzi and C. D'Andrea, "Subspace Tracking and Least Squares Approaches to Channel Estimation in Millimeter Wave Multiuser MIMO," IEEE Transactions on Communications, vol. 67, no. 10, pp. 6766-6780, October 2019.
[9] R. H. Gohary and T. N. Davidson, "Noncoherent MIMO Communication: Grassmannian Constellations and Efficient Detection," IEEE Transactions on Information Theory, vol. 55, no. 3, pp. 1176-1205, March 2009.

[10] L. Zheng and D. N. C. Tse, "Communication on the Grassmann Manifold: A Geometric Approach to the Noncoherent Multiple-Antenna Channel," IEEE Transactions on Information Theory, vol. 48, no. 2, pp. 359-383, February 2002

[11] B. Yang, "Projection Approximation Subspace Tracking," IEEE Transactions on Signal Processing, vol. 43, no. 1, pp. 95-107, January 1995.

[12] K. Ozeki, Theory of Affine Projection Algorithms for Adaptive Filtering, Springer Japan, 2016.

[13] A. A. Ahmadi, A. Olshevsky, P. A. Parrilo, and J. N. Tsitsiklis, "NP-hardness of Deciding Convexity of Quartic Polynomials and Related Problems," Mathematical Programming, vol. 137, no. 1, pp. 453-476, February 2013.

[14] J. Zhang, G. Zhu, R. W. Heath Jr., and K. Huang, "Grassmannian Learning: Embedding Geometry Awareness in Shallow and Deep Learning," 2018.

[15] A. Edelman, T. A. Arias, and S. T. Smith, "The Geometry of Algorithms with Orthogonality Constraints," SIAM Journal on Matrix Analysis and Applications, vol. 20, no. 2, pp. 303-353, 1998.

[16] R. Badeau, K. Abed-Meraim, G. Richard, and B. David, "Sliding Window Orthonormal PAST Algorithm," 2003 IEEE International Conference on Acoustics, Speech, and Signal Processing, 2003. Proceedings. (ICASSP '03)., vol. 5, pp. 261-264, 2003.

[17] X. G. Doukopoulos and G. V. Moustakides, "The Fast Data Projection Method for Stable Subspace Tracking," 2005 13th European Signal Processing Conference, pp. 1-4, 2005.

[18] A. Gonzalez, M. Ferrer, F. Albu, and M. de Diego, "Affine Projection Algorithms: Evolution to Smart and Fast Algorithms and Applications," 2012 Proceedings of the 20th European Signal Processing Conference (EUSIPCO), pp. 19651969, 2012. 